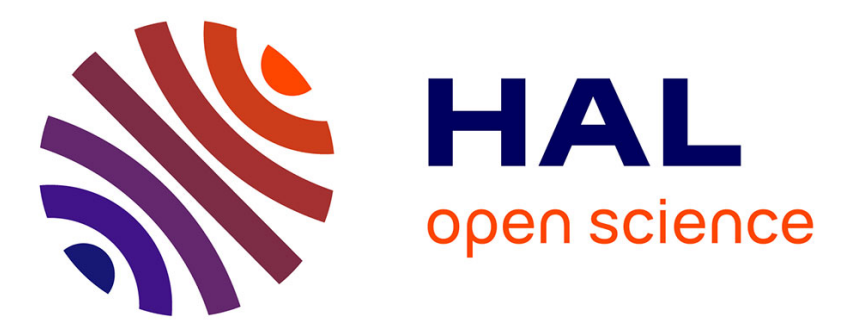

\title{
Comportements de subsistance des hominines au Pléistocène en Afrique et en Europe
}

Jean-Philip Brugal, Olivier Bignon-Lau, Camille Daujeard, Pierre Magniez, Anne-Marie Moigne

\section{- To cite this version:}

Jean-Philip Brugal, Olivier Bignon-Lau, Camille Daujeard, Pierre Magniez, Anne-Marie Moigne. Comportements de subsistance des hominines au Pléistocène en Afrique et en Europe. Jean-Philip Brugal. TaphonomieS, Editions des archives contemporaines; Collection "Sciences Archéologiques", 2017. hal-01744746

\author{
HAL Id: hal-01744746 \\ https://hal.science/hal-01744746
}

Submitted on 27 Mar 2018

HAL is a multi-disciplinary open access archive for the deposit and dissemination of scientific research documents, whether they are published or not. The documents may come from teaching and research institutions in France or abroad, or from public or private research centers.
L'archive ouverte pluridisciplinaire HAL, est destinée au dépôt et à la diffusion de documents scientifiques de niveau recherche, publiés ou non, émanant des établissements d'enseignement et de recherche français ou étrangers, des laboratoires publics ou privés. 


\title{
Comportements de subsistance des hominines au Pléistocène en Afrique et en Europe
}

\author{
Jean-Philip Brugal ${ }^{1}$, Olivier Bignon-Lau ${ }^{2}$, Camille Daujeard ${ }^{3}$, \\ Pierre Magniez $^{1}$, Anne-Marie Moigne ${ }^{3}$ \\ 1 Aix-Marseille Université, CNRS, MCC, UMR 7269 LAMPEA, Aix-en-Provence \\ 2 UMR 7041 ArScAn, Nanterre - CNRS \\ ${ }^{3}$ UMR 7194 HNHP, Paris - Muséum national d'histoire naturelle - \\ CNRS - Université de Perpignan, IPH
}

\section{Introduction}

De nombreuses espèces sont des mangeurs de viande ou d'os, et certaines d'entre elles ont la capacité, et les comportements, de tuer, collecter, transporter ou modifier les ossements de leurs proies. Ils sont parfois accumulés, en grand nombre, dans des lieux plus ou moins spécialisés en terme de reproduction, soins aux petits, consommation de ressources et partage, caches, refuges... Ce sont aussi des lieux d'interactions sociales et de pratiques communautaires importantes.

Parmi ces animaux, les carnassiers et les rapaces, mais aussi des rongeurs (porcépic) ou autres primates [1] montrent de tels comportements, en particulier pour les carnivores sociaux tels que lion, hyène, chien sauvage, loup... considérés comme des «top-predators » dans leur milieu. Il en est de même de l'homme qui a peu à peu, au cours de son évolution, a intégré une part carnée de plus en plus importante dans ses régimes alimentaires.

Qu'est-ce qui distingue les espèces parmi tous ces comportements, et comment l'Homme a développé ses capacités dans une nouvelle 'niche écologique' parmi cette guilde de prédateurs? Quelles sont les stratégies d'acquisition et de consommation des proies, leur sélection et l'importance du transport? Quel est le degré de compétition interspécifique dans cette recherche de subsistance? Comment les hommes ont structuré, en fonction des territoires et des ressources, leurs modes de vie et leurs innovations techniques? 
Les sites archéologiques, en cavités ou en plein-air (souvent en bordure de cours d'eau), recèlent des assemblages composés d'industries sur pierre et, si les conditions taphonomiques le permettent, des restes fossiles osseux et dentaires; l'ensemble pouvant présenter une structuration spatiale, avec pour des périodes « récentes » l'existence de foyers, et de zones de concentration préférentielle résultant de la gestion des activités quotidiennes. Les études paléontologique, taphonomique et archéozoologique permettent alors de dresser plus précisément l'origine de ces accumulations et de mieux connaitre les pratiques socio-économiques des espèces humaines qui se sont succédées depuis plus de 3 millions d'années (Ma).

L'apport des études taphonomiques dans l'appréhension de ces accumulations osseuses est primordial, permettant d'identifier et de préciser les comportements propres à notre lignée. Dans les chapitres qui suivent, quelques illustrations de ces problématiques seront données dans le cadre chronologique du Paléolithique, depuis le plus ancien en Afrique, jusqu'au plus récent en Europe.

\section{Hominines du Plio-Pléistocène d'Afrique (J.-Ph. B.)}

Les plus anciens hominines se trouvent en Afrique avec une grande diversité de taxons : depuis le genre Ardipithecus (5 Ma), jusqu'aux genres Australopithecus, Paranthropus, Kenyanthropus à partir de 4 Ma... et Homo. Le plus vieux représentant de notre genre provient d'un site éthiopien daté de $2.8 \mathrm{Ma}[2]$ et seule une poignée de sites archéologiques sont datés de plus de 2 Ma en Éthiopie et au Kenya en particulier [3]. Le plus vieux site livrant des outillages en pierre est daté de près de 3.3 Ma dans la région de l'Ouest Turkana, au Nord Kenya [4]. Appelée Lomekwien, cette culture précède celles de l'Oldowayen (2.6-1.8 Ma) suivi par l'Acheuléen (e. 1,8 Ma).

L'acquisition et la consommation de produits carnés, ou carnivorie, sont un facteur crucial dans l'évolution humaine. Il a entrainé d'importantes adaptations physiologiques, métaboliques, morphologiques, écologiques et éthologiques. Il est donc intéressant d'en connaitre l'origine et, l'apport des études taphonomiques dans la formation des concentrations fossiles et leurs associations avec des restes humains et/ou des artefacts lithiques a été prépondérant (par ex. pour historique : travaux de Henri-Martin, Breuil, Pei, Hughes in [5]).

Suite au débat sur l'origine des dépôts fossilifères dans les grottes d'Afrique du Sud, et du rôle des australopithèques dans ceux-ci, l'analyse taphonomique détaillée des ensembles osseux (fractures, traces, etc.) a permis de meilleures reconstitutions. Présentés à l'origine comme « l'Homme chasseur» tuant et accumulant les restes de ses proies, l'étude comparative de ces ensembles avec ceux d'autres carnivores a permis en fait de montrer qu'il s'agissait de l'inverse : les hominines ayant été les proies des léopards en particulier [6: Figure 1]. Outre ce travail pionnier en taphonomie on peut également citer l'ouvrage de Behrensmeyer et Hill, au titre évocateur : Fossils in the Making [7]. Ces travaux, et beaucoup d'autres depuis, concernent les gisements pliopléistocènes est- et sud-africains, complétés par l'établissement de référentiels actuels, portant sur les milieux naturels ainsi que sur les sociétés humaines traditionnelles (ex. Nunamiuts, Bushman, Hottentots). Ils ont également démontré le rôle important joué par d'autres prédateurs non-humains dans les accumulations osseuses, en cavités ou 


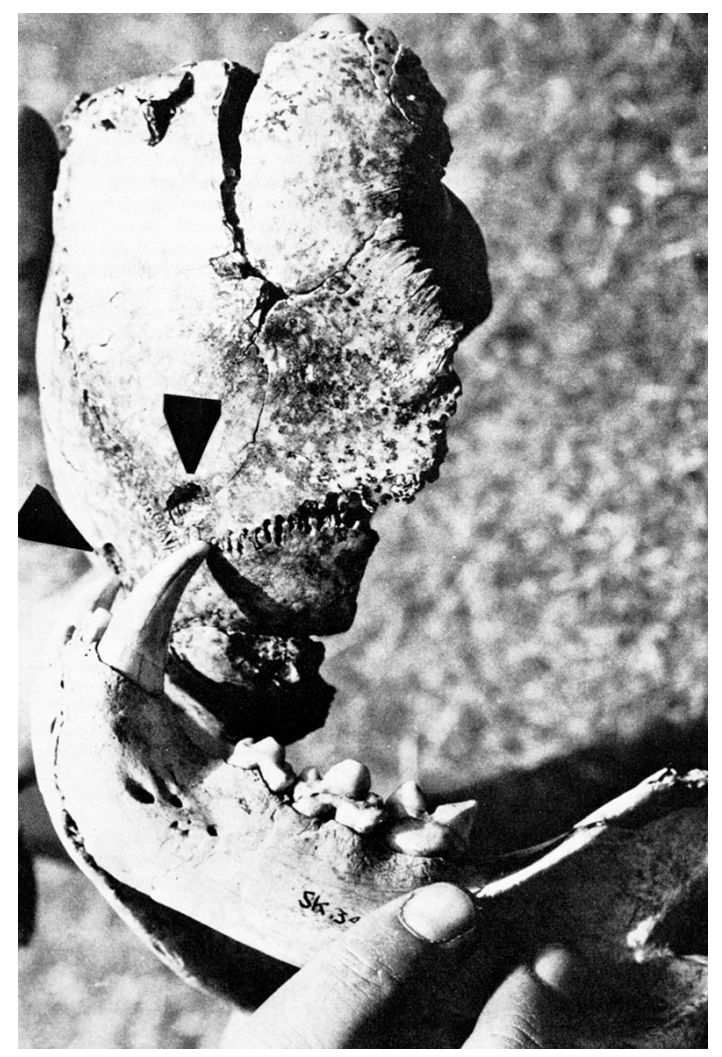

Figure 1 - Calotte crânienne avec marques de léopard (modifié de [6]).

en plein-air, ainsi que celui des groupes humains, et ont participé au débat encore très actuel sur les modes d'acquisition - chasse ou charognage.

Dans le contexte des gisements plio-pléistocène du rift est-africain, de nombreuses recherches ont été initiées à partir des sites archéologiques de plein-air de Tanzanie et du Kenya. Une typologie des gisements, fondée sur le degré et la densité des restes lithique et osseux, a été proposée [8, 9, 10 et voir 11,12]. Le concept de « central-place foraging »(lieu-focus de consommation) a été depuis largement complété par des analyses plus précises sur les espèces exploitées (taille des proies, courbe de mortalité), les éléments osseux retrouvés (ex. os riche en viande comme le fémur vs os pauvre comme les métapodes) et leur degré de fracturation, la conservation différentielle des restes, ou les marques laissées sur les os par des tranchants lithiques [e.g. 13]. Dans les écosystèmes plio-pléistocènes africains, la guilde des grands carnivores est très diversifiée (incluant les félidés à dents de sabre) et leurs traces sur les ossements (rognage, sillons dentaires, impacts) sont tout autant considérées, tout comme la présence de coprolithes. Dans ce contexte, il est donc déterminant de connaître les paléoenvironnements et les conditions de dépôts. 


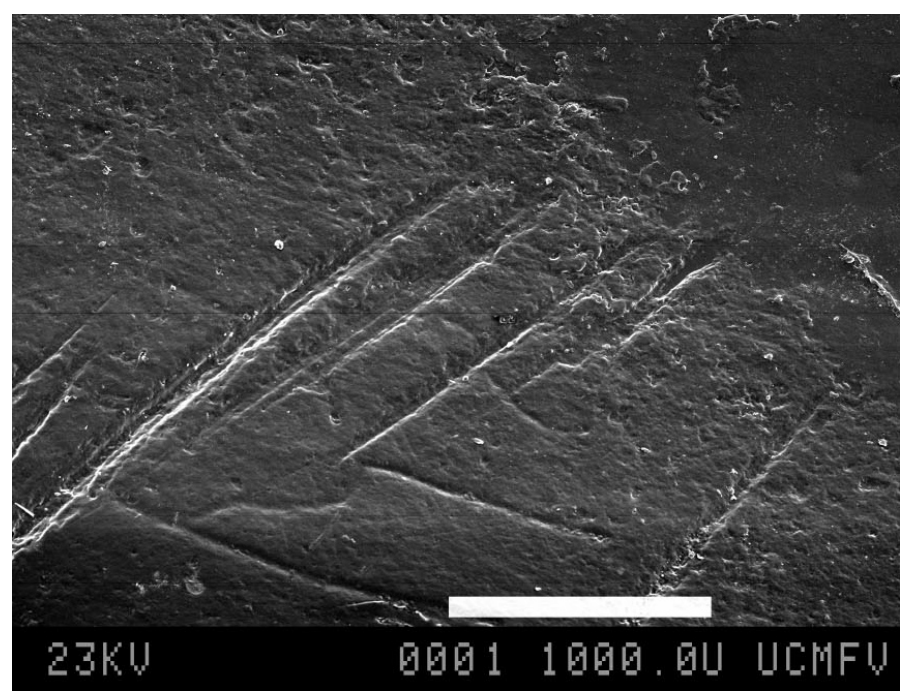

Figure 2 - Stries de découpe anthropique sur un os du site éthiopien de Gona, daté d'e. 2.6 Ma [15] (macrophoto).

Pour les périodes anciennes $(<2 \mathrm{Ma})$, les comportements humains de subsistance sont encore mal documentées. Deux ossements trouvés en surface, datés à e. $3.4 \mathrm{Ma}$ (Dikika, Éthiopie) porteraient des stries de découpe [14], ainsi que d'autres marques (piétinement). Des preuves plus évidentes proviennent des sites de Gona en Éthiopie, où, dès 2.6 Ma, des stries sur les ossements (Figure 2) apparaissent sans ambiguïté [15]. Les sites de Lokalalei de la formation de Nachukui (Ouest Turkana) sont datés entre 2.3-2.4 Ma et livrent également quelques indices d'exploitation de carcasses, en bordure de rivières ou de lacs [16]. Avec une faune très diversifiée (env. 55 taxa, dont des reptiles, oiseaux, rongeurs, carnivores, primates, équidés et de nombreuses antilopes ; [17, 18]), Lokalalei 1 (La1) correspond à une zone relativement boisée procurant ombre, protection, ressources végétales et eau potable. Si l'accumulation est en partie naturelle, d'autres agents biologiques sont impliqués. Quelques rares stries et actions sur les ossements de bovidés de taille 2 (ex. gazelle) et 3 (ex. gnou), mais aussi sur une carapace de tortue indiquent des activités opportunistes (charognage passif). On note aussi l'action réduite de carnivores prédateurs ayant détruit une partie des ossements. Pour ces périodes, la consommation de viande était encore très limitée, complétée par une part végétale plus conséquente.

Un autre exemple concerne le site oldowayen de Kanjera (Sud Kenya), daté d'e. 2.0 Ma, ayant livré des arguments clairs d'une carnivorie déjà plus persistante [19]. Les groupes humains se sont succédés au cours du temps, à la même place, et ont chassé et consommé des carcasses de petits ongulés presque complètes, avec parfois des accès occasionnels à de plus grands gibiers (taille 3) par charognage. Non seulement la viande, mais d'autres produits étaient consommés (viscères, moelle, cervelle). Dans d'autres régions (Est Turkana), des indices d'un élargissement de la diète des hominines vers des petits animaux et des animaux aquatiques [20] ont été signalés. 
Le complexe de gisements d'Olduvai (Tanzanie) découverts et fouillés d'abord par L. Leakey (à partir de 1931) reste emblématique des recherches en Afrique de l'Est, foisonnant d'analyses sur les paléoenvironnements, la taphonomie des sites et leur interprétations palethnologiques (e.g., [21, 22, 23, 24]). Le plus important, FLK22 (FLK Zinj, Bed I), daté de 1.8 Ma et localisé près d'un lac dans une zone avec des arbres, buissons proche de plaines, a livré une impressionnante accumulation osseuse et des artefacts lithiques. Les stries et les marques de percussion sont abondantes, y compris sur les os riches en viande (humérus, fémur), ainsi que les marques de carnivores et de crocodile. Près de 14 taxons de toutes tailles (gazelles, impalas, suidés, équidés, pachydermes) montrent des stries de boucherie [3]. Si la plupart des sites d'Olduvai ne sont que des associations fortuites d'outillages et d'ossements, FLK Zinj est une preuve forte d'un changement du rôle écologique des hommes, avec une plus forte probabilité pour une capacité de chasse, mais aussi de charognage plus actif (« confrontational scavenging 》) [24].

Il s'agit là des premières étapes reconnues en Afrique de l'Est de l'émergence de la carnivorie chez les premiers hommes. Elles s'accompagnent de changements profonds qui se produisent alors, notamment dans la diversité des outillages lithiques. Nous n'avons évoqué que l'Oldowayen, mais les sites de l'Acheuléen, très nombreux du Nord au Sud du continent, témoignent aussi du développement graduel des capacités cynégétiques des groupes humains, sans pour autant abandonner les pratiques de récupération plus opportunistes (charognage, collecte).

\section{Hominines du Pléistocène d'Eurasie}

\subsection{Le Paléolithique inférieur (A.-M. M.)}

À partir de 1,8 Ma d'années, les outillages de pierre associés à des fossiles de grands mammifères deviennent plus abondants, en Afrique, comme on vient de le voir, mais également en Asie (ex. Longgupo, [25] et aux portes de l'Europe (Dmanisi, [26]). Il s'agit toujours de sites de plein-air et le plus souvent d'accumulations de type catastrophique (inondation, épisodes volcano-sédimentaires) indiquant que les hommes ont profité, comme les grands carnivores d'amoncellements, de carcasses. Les stries sur les ossements ainsi que la fracturation des diaphyses d'os long sont rares mais représentent les seules preuves d'activités anthropiques et de l'utilisation des galets aménagés ou éclats de taille associés.

À partir de 1,5 Ma jusqu'à 800000 ans, plusieurs exemples de sites de boucherie sont observés en Europe occidentale (Espagne, Italie, Allemagne, France, [27, 28]) au sein d'accumulations d'origine fluviatile ou lacustre. Elles ressemblent beaucoup à celles décrites en Afrique (Kenya, Tanzanie). Les hommes s'installent en groupes familiaux comme peuvent en témoigner des dents de lait découvertes dans certains sites d'habitat (Barranco Leon, Sud Espagne). Ils débitent sur place les galets de rivière pour exploiter des carcasses de grands mammifères de taille moyenne à grande. Les rares stries de découpe sont décrites sur des ossements de cervidés, très abondants (Cervus, Megaceroides), de grands bovidés, d'hippopotames, assez abondants à ces périodes et de Mammuthus meridionalis. Tous ces ossements présentent aussi de nombreuses 
traces de grands carnivores (Canis mosbachensis, Panthera gombaszogensis et Pachycrocuta brevirostris).

Un deuxième type de site est, comme en Afrique, représenté par l'association de carcasses isolées de grands à très grands herbivores (proboscidiens, hippopotames) avec des outils lithiques, galets aménagés, grands éclats, débris de débitage, toujours d'origine locale; ces lieux peuvent également livrer des coprolithes de prédateurs non humains. Les Hommes profitent de carcasses mortes naturellement ou tuées par les grands carnivores (Fuente Nueva 3, Espagne, [29]). La question de la succession des intervenants sur ces sites se pose et nécessite une étude taphonomique précise, sur la nature des traces et leurs auteurs respectifs, en comparant aux référentiels expérimentaux. La dimension spatiale et l'analyse géomorphologique de tels sites participent aux interprétations. Les résultats montrent que les carnivores ont eu un accès primaire à la carcasse et qu'ils sont le plus souvent à l'origine de l'accumulation (prédation).

Les premières occupations en grottes sont connues en Europe de l'Ouest (ex. Atapuerca en Espagne - [30] -, ou Le Vallonnet en France), et les hommes n'hésitent pas à s'introduire dans des cavités servant de repaires de grands carnivores (hyénidés, félidés et canidés). Les outils sont rares mais témoignent, comme la présence de restes humains ou de traces observées sur les os d'animaux, du passage furtif des hommes dans ces milieux. L'existence de gisements à faible indice de fréquentation humaine est alors posée [31] témoignant d'un nouveau comportement de récupération de ressources animales. Cette stratégie va se poursuivre jusque durant le Paléolithique moyen.

En Eurasie, ce n'est qu'à partir de 800000 ans, que l'on peut observer dans plusieurs sites, des accumulations attribuées en partie aux groupes humains. En effet, les espèces animales sont plus variées et leurs profils de mortalité ne correspondent pas à un profil type prédation de grand carnivores (jeunes et âgés) ni à des accumulations de type catastrophique (jeunes à âgés décroissants), avec plus d'adultes représentés. Les éléments squelettiques sont sélectionnés, et les stries et la fracturation intentionnelle beaucoup plus abondantes, tout comme la proportion de l'outillage. Les restes humains sont en général présents et peuvent porter des traces de cannibalisme comme à Atapuerca (TD 6) en Espagne [32].

Cette tendance se précise tant en grottes qu'en plein-air avec le phénomène acheuléen, en Asie (Yunxian, Zhoukoudian, Chine, Ngebung, Sangiran, Indonésie [33]), au Proche-Orient (ex. Gesher Benot Ya'aqov, [34]) et en Europe où les témoins anthropiques sont associés à des accumulations naturelles ou à des proies de grands carnivores (Cagny-L'Épinette, Orgnac 3-c7, Isernia La Pineta [Figure 2], sites du Latium; [35, 36]). Les traces sur les ossements sont abondantes, correspondant à du dépeçage, de la désarticulation avec une fracturation intentionnelle des os longs, des mandibules et des crânes. La recherche de diaphyses d'os longs et de défenses de proboscidiens est signalée, servant à préparer des outils comme les bifaces ou de grands éclats (Fontana Ranuccio, Ngebung, Dingsun). On observe aussi des traces sur les extrémités de pattes de carnivore (ours, lynx), démontrant l'exploitation de fourrures. Globalement, l'homme a accès rapidement aux carcasses, pratiquant un charognage actif, avec peut-être des débuts de chasse. 


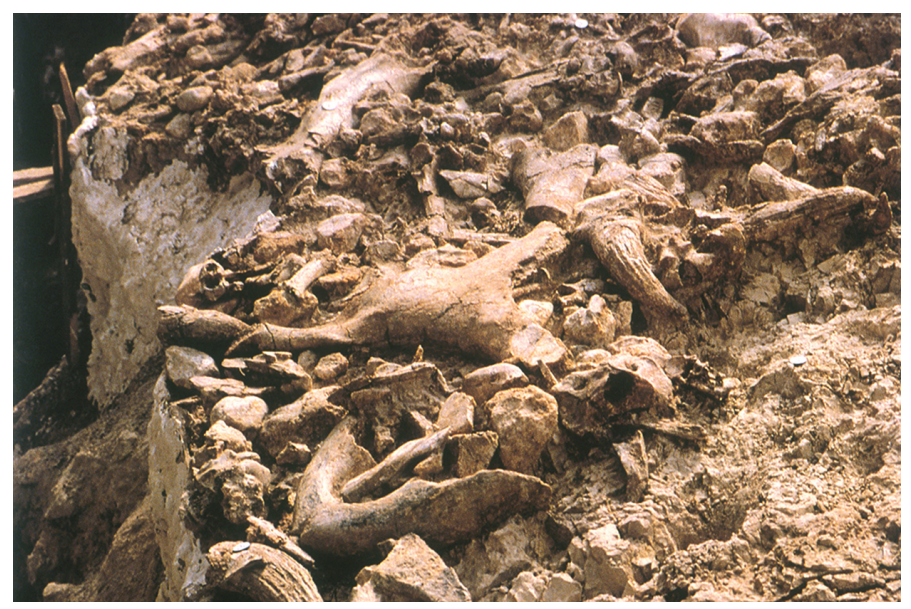

Figure 3 - Isernia La Pineta, Molise, Italie (détail d'un niveau riche en bisons). (Cliché A.-M. Moigne.)

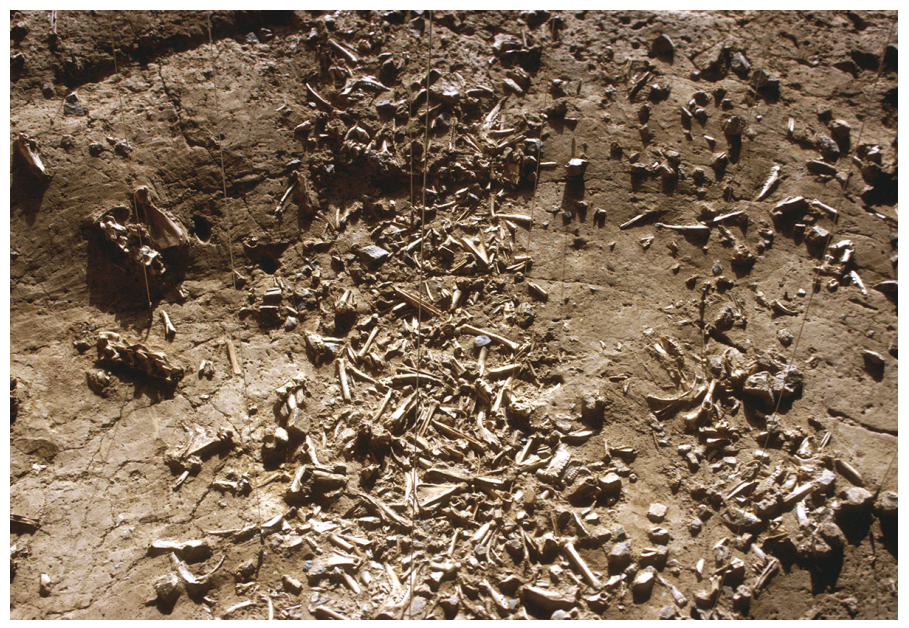

Figure 4 - La Caune de l'Arago, Pyrénées Orientales, France (détail du niveau L). (Cliché A.-M. Moigne.)

Plusieurs sites indiquent en effet la chasse comme mode principal d'approvisionnement des groupes humains (La Caune de l'Arago [37], Orgnac 3, Terra Amata). Les espèces animales sont choisies dans différents biotopes et les animaux accumulés sur les sites présentent des critères de sélection par rapport au sexe et à l'âge. Les espèces privilégiées sont les chevaux, les grands et petits bovidés (bison, aurochs, mouflon, thar), les cervidés (cerf, daim, ou renne). Les très grands herbivores (mammouth ou rhinocéros de prairie) sont présents mais considérés comme espèces complémentaires, c'est-à-dire peu ou pas chassées mais peut être recherchées et charognées pour des raisons dépassant le simple phénomène alimentaire. La proportion de carnivores sur ces sites est très faible. Le plus souvent les carcasses sont désarticulées et la fracturation 
des os est systématique, voire standardisée (récupération de la moelle). Les sites sont aussi choisis en fonction de la matière première lithique disponible et de la situation $\mathrm{du}$ lieu pour l'approvisionnement en viande, alors crucial dans le contexte des grandes glaciations du Pléistocène moyen.

Les sites préhistoriques peuvent être classés selon la durée d'occupation et la saisonnalité déduite des jeunes animaux. En effet d'après le stade de remplacement dentaire des jeunes et d'usure sur les adultes, il est possible d'évaluer la période de l'année durant laquelle tous ces animaux ont été transportés sur le site. Les résultats de l'étude archéozoologique croisés avec ceux des approvisionnements en roche (silex, quartz, quartzite...), qui peuvent être assez éloignés (40 km), permettent de préciser la fonction des sites : semi-permanents, saisonniers ou haltes de chasse. Sur les sites d' « ateliers de taille », les ossements sont plus rares, ou absents, et peuvent ressembler à des accumulations de type secondaire.

\subsection{Le Paléolithique moyen d'Europe occidentale}

\subsubsection{Le Paléolithique moyen ancien (C. D.)}

Le Paléolithique moyen ancien débute en Europe occidentale dans la seconde moitié du Pléistocène moyen, aux environs de 400000 ans (stades 11 à 6 dans la chronologie isotopique). Au cours de la première période, les hominines sont des Homo heidelbergensis, dont le fossile type a été découvert près de Heidelberg en Allemagne. Le site de la Sima de Los Huesos à Atapuerca en Espagne a livré de très nombreux restes humains de cette période (au moins 28 individus) datés de plus de 300000 ans. Ces fossiles indiquent un degré d'encéphalisation et une dérive génétique qui ont pu mener à la grande dextérité observée chez les Néandertaliens « classiques » plus récents, que ce soit dans la taille d'outils, la chasse ou encore dans le traitement boucher des carcasses $[38,39]$.

L'Europe du Nord connaît à cette période l'installation de la steppe à mammouth (Mammuthus trongontherii) favorable au développement de grands troupeaux d'herbivores [40]. Ce biome va perdurer et on le retrouve également pendant le Pléistocène supérieur ( $c f$. infra). Les premières incursions des populations humaines en Europe du Nord ont été alors favorisées par la présence de ces nombreuses proies et par les niches écologiques laissées vacantes par les hyper-prédateurs. Félins à dent de sabre et hyènes géantes, pourvoyeurs de charognes, disparaissent en effet peu à peu pour laisser la place à d'autres carnivores plus « modernes » comme les hyénidés et les canidés [41].

Cette période du Paléolithique moyen s'accompagne de nouveaux comportements qui vont contribuer à faciliter l'implantation humaine à la fois dans le Sud comme dans le Nord de l'Europe. Les groupes humains développent alors nettement leurs capacités cynégétiques, dans un cadre technique et coopératif maîtrisé. Les premières armes de chasse (épieux en bois) sont découvertes en Europe, à Schöningen en Allemagne [42], ou encore à Clacton-on-Sea en Grande-Bretagne [43], et datent d'environ 350000 ans. Bien que la chasse aux grands herbivores ait été attestée dans des assemblages fauniques anciens d'Afrique de l'Est (cf. supra) ou du Levant (ex. Ubeidiya : [44]), les témoins de chasses sélectives et organisées se multiplient à partir de cette période 
en Europe, toujours en parallèle aux activités de charognage. En France, des séries lithiques évoquent la mise en place de nouvelles techniques de taille standardisées et prédéterminées (débitage Levallois) dès 350000 ans (Orgnac 3 - 4 à 1 : [45]; SudOuest de la France : [46]). La domestication du feu, qui se généralise entre 400000 et 300000 ans [47], permettra, grâce à la cuisson des aliments, un gain d'énergie considérable et de meilleurs moyens de défense face aux carnivores présents dans le territoire, tout du moins dans les sites d'habitat. En effet, à partir de l'OIS 11 (env. 400000 ans), la plupart des sites présentent des traces de chauffe sur les ossements. Plusieurs concentrations ont d'ailleurs été observées et étudiées lors de fouilles, sous forme de foyers ou de niveaux cendreux (Menez Dregan, Orgnac 3, Terra Amata, etc.). Ces gisements permettent d'intégrer plus précisément la notion d'habitat, et les occupations humaines peuvent être assimilées à des campements structurés, le plus souvent réoccupés à plusieurs reprises pour des activités similaires, pouvant créer là aussi, selon la nature des sédiments, des palimpsestes.

À Biache-Saint-Vaast, dans le Nord de la France, aux environs de 250000 ans, des pré-néandertaliens ont chassé et exploité pour leur viande et leur peau l'ours brun de façon régulière, aux côtés de l'aurochs, du rhinocéros de prairie (Dicerorhinus hemitoechus), du cheval et du cerf [48]. Au Lazaret (Alpes-Maritimes), à la même période, ce sont les cerfs et les bouquetins qui ont été les proies préférentielles [49]. Dans le site de La Borde (Lot), les hommes ont tiré parti d'un ancien aven aujourd'hui comblé pour exploiter l'aurochs de façon sélective et organisée [50]. Ils ont fait de même pour le Bison, comme à Coudoulous I (Lot) daté d'env. 160000 ans [51]. Les hommes s'adaptent et orientent donc leurs stratégies selon les topographies et les environnements. Ils exploitent les espèces locales, qu'elles soient grégaires ou solitaires, grâce à diverses techniques de chasse (affût, poursuite, approche, battue, piégeage).

Les sites en grotte comme ceux de plein-air sont visités en alternance par les hommes et les carnivores. L'exemple des sites karstiques de Gran Dolina TD10-1 [52] et de Galeria [53] en Espagne ou de Payre dans le Sud-Est de la France [54, 55] attestent de cette proximité entre hommes et carnivores : utilisation alternée des sites comme lieu d'habitat, d'hivernation ou de repaire; confrontation directe entre prédateurs ou charognage des restes abandonnés par les divers occupants [56]. Quelques ossements représentent des témoins encore plus directs de ces interactions. Des restes de lion des cavernes provenant de Gran Dolina TD10 témoignent de la récupération de la peau, de la viande et de la moelle par les hommes [57]. Au Lazaret, dans le Sud-Est de la France, une mandibule de lynx des cavernes présente des marques de dépouillement [58]. Enfin, plus au Nord, à Schöningen, un humérus d'Homotherium a été utilisé pour retoucher des tranchants d'outils lithiques [59].

Les herbivores de taille moyenne à grande (bisons, chevaux, cerfs, bouquetins, etc.) dominent les listes fauniques de cette période. Néanmoins les pachydermes constituent encore un apport important en protéines animales. Les sites du Latium en Italie ou d'Áridos, de Torralba et d'Ambrona en Espagne, datés entre 400000 et 250000 ans, ont livré d'importantes accumulations d'ossements d'éléphant (Palaeoloxodon antiquus) morts naturellement sur les rives d'anciens fleuves et exploités par les groupes humains [60-62]. L'exploitation de la mégafaune est également attestée dans des sites 
plus récents, ex. La Cotte Saint Brelade à Jersey pour le mammouth (Mammuthus primigenius) et le rhinocéros laineux (Coelodonta antiquitatis) [63], ou encore Payre pour les rhinocéros de Merck et de prairie (D. mercki et D. hemitoechus) $[54,55]$.

De nombreux sites attestent du perfectionnement des techniques d'acquisition (transport et traitement des meilleurs morceaux au camp) et de consommation (exploitation systématisée et intensive) des carcasses animales. À l'image du site de Qesem (niveaux du Paléolithique moyen) au Proche-Orient [64], le site d'Orgnac 3 dans la vallée du Rhône, daté d'environ 300000 ans, voit apparaître dans le haut de sa séquence une standardisation des marques de boucherie [45].

De nouveaux comportements de subsistance se généralisent donc dans la deuxième moitié du Pléistocène moyen, ainsi que bon nombre d'innovations techniques performantes. Parallèlement à ces acquis, la densité humaine, la complexité de réseaux sociaux resserrés et la viabilité des nouvelles niches écologiques ont également dû jouer un rôle fondamental dans l'implantation humaine et la transmission culturelle à grande échelle $[65,66]$.

\subsubsection{Le Paléolithique moyen récent (P. M.)}

En Europe occidentale, le paléolithique moyen récent peut être défini entre 130 et 35 ka BP, couvrant une grande partie du Pléistocène supérieur (stades 5 à 3 de la chronologie isotopique). L'optimum climatique autour de 130 ka renvoie à un climat tempéré et humide. L'ambiance est plus fraiche jusqu'à 70 ka et nettement froide et sèche entre 70 et $60 \mathrm{ka}$ (stade 4). Entre 60 et 35 ka le climat est plutôt froid et humide mais la période est marquée par des oscillations climatiques courtes et brusques (évènements d'Heinrich par ex, voir infra), parfois de fortes amplitudes, qui témoignent d'intervalles climatiques frais et humides à très froids et secs. Ces variations climatiques ont eu un impact sur les paléoenvironnements, sur la biocénose notamment, et par extension sur les modes de vie des groupes humains occupant ce vaste territoire : les Néandertaliens (Homo neanderthalensis).

Dans les années 1980, le comportement alimentaire des Néandertaliens était qualifié d'opportuniste, se concentrant surtout sur des gibiers de taille moyenne, avec une très faible contribution aux proies de grande taille, en partie acquis par charognage [66, 67]. Les développements méthodologiques et une meilleure interprétation des données, la multiplication des analyses archéozoologiques et taphonomiques, permettent de brosser un tout autre scénario.

Les comportements alimentaires et les stratégies d'acquisition des ressources carnées pratiquées par les Néandertaliens n'étaient pas stéréotypés, et ils semblent qu'ils étaient des chasseurs efficaces. Les recherches révèlent des modèles variés, parfois complexes avec une utilisation raisonnée de la topographie et une mobilité importante. La représentation d'herbivores dans les sites est non seulement fonction de choix anthropique mais aussi de paramètres environnementaux modulant les aires de répartition spécifiques et leurs densités.

Les études menées dans la vallée du Rhône illustrent bien la faculté de l'Homme de Néandertal à occuper des lieux stratégiques pour exploiter divers ongulés à différentes 
saisons de l'année suivant l'abondance des ressources, exprimant des occupations brèves mais aussi de plus longue durée [68]. En contexte topographique contrasté, à proximité de plaines ou de plateaux, une majeure partie de leur alimentation carnée provenait des troupes d'équidés, de bovinés et de cervidés reflétant une grande variabilité dans les territoires de chasse exploités comme dans les stratégies de transport des carcasses mises en œuvre [e.g., 69-76]. En contexte rocheux, bouquetins et chamois prennent le relais [77].

De plus, les Néandertaliens ont la capacité à conduire des chasses orientées sur certaines espèces, voire devenir très spécialisés, lors d'occupations brèves. Par exemple, le site des Pradelles (Sud-Ouest), daté de l'OIS 3, présente des ensembles dominés par le renne. Les hommes ont chassé collectivement un grand nombre d'individus de tous âges à la fin de l'été et au début de l'automne [78, 79]. Beaucoup de restes d'os longs des membres sont présents. Les études taphonomiques suggèrent qu'il s'agit d'un site de boucherie secondaire, dans lequel les éléments les plus nutritifs ont été transportés. Le traitement systématique et standardisé des carcasses pourrait également renvoyer à une activité de stockage des ressources ou, a minima, d'un transport secondaire de la matière carnée pour une consommation différée dans un camp de base [78, 79]. D'autres sites partagent la plupart de ces caractéristiques comme SalzgitterLebenstedt, en Allemagne [80] ou Jonzac [81], avec le renne comme proie dominante, ou encore Mauran [82] avec le bison. Ce site, au pied des Pyrénées, s'illustre par une représentation quasi exclusive de bison, au moins 137 individus provenant d'une petite surface fouillée d'un gisement bien plus vaste [83]. La chasse s'est étalée là aussi de la fin de l'été au début de l'automne, ciblant préférentiellement les femelles accompagnées des jeunes.

Néandertal ne se cantonnait cependant pas aux grandes proies et le petit gibier (lapins, oiseaux...) était également exploité, pour consommation ou autres utilisations [e.g., 73, 84-86] Par exemple, dans la couche 4 des Canalettes, l'étude taphonomique démontre clairement l'acquisition du lapin [87], et certains chercheurs parlent de l'utilisation de plumes de rapaces (coiffe?).

Les analyses taphonomiques dans ces études sont fondamentales, en particulier pour déterminer si l'homme était l'agent principal des accumulations [88-91]. Ce, d'autant plus que de nombreux sites de cette période montrent une représentation non négligeable des carnivores (en termes de nombres de restes et/ou d'individus), suggérant un impact significatif sur les ensembles osseux, parfois même en tant qu'accumulateur principal. Ainsi, de nombreux ensembles présentent cette double origine homme / carnivore; avec le cas particulier des occupations d'ours des cavernes pour hivernation [92].

Nos données sur la subsistance des Néandertaliens suggèrent une grande mobilité des groupes au sein de territoires plutôt restreints $(\approx 100 \mathrm{~km})$, variable selon les saisons et la disponibilité des ressources, dans un contexte de faible densité humaine. Les stratégies d'acquisition et de traitement des ressources animales sont variables, relativement diversifiées, et parfois complexes, associées à un équipement technique élaboré. Si la chasse devient dominante, individuelle à collective, d'autres techniques perdurent en parallèle (charognage, collecte). Les néandertaliens exploitaient tout ou 


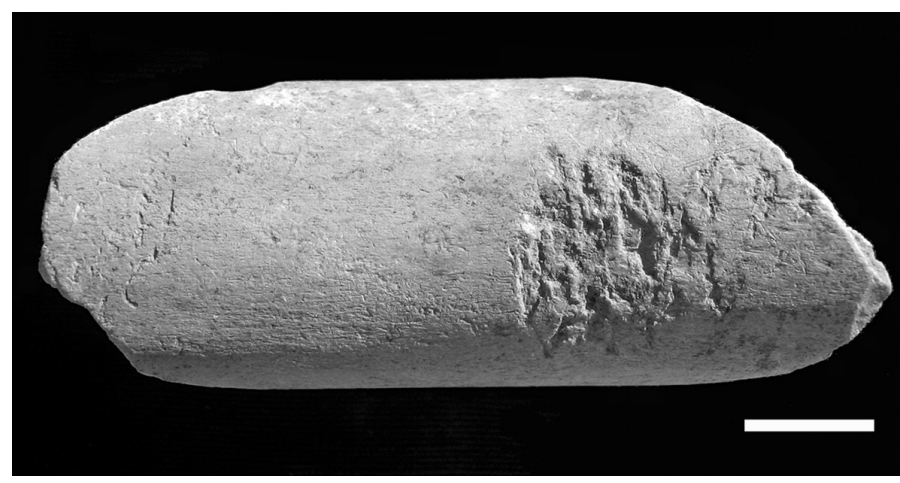

Figure 5 - Fragment diaphysaire d'os long de moyen herbivore présentant une plage de retouchoir (abri du Maras, fouilles R. Gilles, couche 5, E3-8722, musée de la Préhistoire d'Orgnac). (Cliché C. Daujeard.)

partie des carcasses (viande, moelle, viscères, graisse, os, dents... ) d'un large spectre de proies, à des fins alimentaires (avec probablement des premiers essais de stockage) mais aussi utilitaires (retouchoirs, fig.) ou symboliques (outillages, parures...) [e.g., 93, 94]. Ces groupes humains avaient une organisation sociale structurée et pouvait élaborer une gestion / planification temporelle de leurs activités, à l'instar des populations du Paléolithique supérieur.

\subsubsection{Le Paléolithique supérieur (O. B.-L.)}

Le Paléolithique supérieur (40-10 ka, fin stades 3 et 2 de la chronologie isotopique) est jalonné de plusieurs épisodes de grands froids correspondant notamment aux événements de Heinrich 4 à $1^{1}$, un degré d'englacement maximal du Dernier maximum glaciaire (20-15 ka) et l'interstade Bölling-Alleröd au cours du Tardiglaciaire [95, 96]. Cette instabilité climatique est contemporaine de nombreuses cultures préhistoriques dont l'expansion pan-européenne souligne la forte interconnexion socioéconomique. Ces grandes entités culturelles telles que l'Aurignacien, le Gravettien, le Solutréen ou le Magdalénien, sont le fait des hommes anatomiquement modernes (Homo sapiens sapiens), généralement définis par leurs industries lithiques et osseuses. Toutefois, le passage du Paléolithique moyen au Paléolithique supérieur est l'objet de débats car le scénario d'une supplantation biologique et culturelle synchrone et homogène, c'est-à-dire le remplacement des techno-complexes moustériens des Néandertaliens (Homo sapiens neanderthalensis) par les sociétés aurignaciennes des Hommes modernes, n'apparaît plus aussi évident en l'état actuel des recherches. En différents lieux géographiques, des « industries de transition » comme le Châtelperronien et le Proto-Aurignacien sont distinguées sans que leurs auteurs soient clairement établis [97-100]. Ces débats cherchant à comprendre les rythmes et mécanismes des changements culturels, se heurtent cependant à des positionnements épistémologiques antagonistes, mais surtout à des données très lacunaires [101]. Pour cette raison, les

1. Les événements de Heinrich témoignent de l'instabilité des calottes polaires et des glaciers qui couvraient alors une partie des continents de l'Hémisphère nord. Ils renvoient aux débâcles massives d'icebergs dans l'océan Atlantique Nord qui ont ralenties la circulation thermohaline, entraînant plusieurs refroidissements climatiques. 
considérations développées ci-après ne concernent que les sociétés appartenant sans conteste au Paléolithique supérieur, soit à partir du début de l'Aurignacien (après $35000 \mathrm{BP})$.

Les communautés biotiques du Paléolithique supérieur sont généralement désignées par le terme de «steppe à mammouth » [102]. À l'échelle de toute l'Eurasie, quatre espèces sont systématiquement observées : le mammouth (Mammuthus primigenius), le cheval (Equus caballus), le bison (Bos priscus), et le renne (Rangifer tarandus). Cependant, les sites archéologiques démontrent une diversité animale beaucoup plus grande, révélant l'existence d'un paysage en mosaïque sans analogues modernes. L'examen d'associations fauniques provenant de sites naturels, sans intervention humaine (ex. aven-pièges), confirme cette plus large diversité, tant en ongulés qu'en carnivores [103].

La présence conjointe de nombreuses espèces d'herbivores au sein de la steppe à mammouth témoigne d'une structuration complexe et variable des ressources alimentaires dans l'espace. Espèces généralistes (et monogastriques), le mammouth et le cheval captent alors l'essentiel des flux d'énergie des écosystèmes et elles sont des espècesclés au sens où elles ont un impact prépondérant sur l'ensemble des peuplements écologiques et la formation des paysages. Les espèces animales dites 《redondantes », très généralement des ruminants, ont une influence moindre parce qu'elles occupent des niches écologiques spécialisées. Néanmoins, elles traduisent la richesse de la biodiversité et cette mosaïque des paysages qui a fait l'objet d'acquisition directe, indirecte ou par piégeage par les diverses sociétés humaines du Paléolithique supérieur.

$\mathrm{Au}$ cours du Paléolithique supérieur, l'instabilité climatique n'a qu'assez peu modifié la relative stabilité des peuplements animaux européens. Néanmoins, au cours de l'interstade Bölling-Alleröd, vers 12000 ans BP, on assiste à la désintégration des communautés animales de type steppe à mammouth en Europe de l'Ouest avec de nombreuses disparitions (mammouth, rhinocéros laineux, mégacéros, lion des cavernes, etc.) et à des modifications d'aire de distribution topographique (bouquetin, chamois) ou géographiques (renne, bison, bœuf musqué, antilope saïga, etc.). Les chasses menées sur les espèces-clés ont peut-être conduit les hommes à devenir un facteur aggravant de la disparition de la steppe à mammouth en Europe [104].

En Europe occidentale, l'Aurignacien va perdurer d'environ 35000 à 28000 ans BP sur de vastes territoires, de l'Autriche à l'Espagne et de la Belgique au nord de l'Italie. À la période de l'Aurignacien ancien, ces sociétés ont su tirer avantage des potentialités des territoires comme l'atteste l'exploitation des gibiers. Ainsi, il est possible de réfuter l'hypothèse de chasses spécialisées exclusivement au renne, car même si ce gibier fut l'une des principales proies, il est souvent accompagné ou dépassé par d'autres comme le cerf, le cheval ou le bison [105]. La recherche d'apports en viandes et en graisse détermine les choix cynégétiques variables, même dans des régions connexes comme entre le Bassin aquitain, le Pays Basque et les Cantabres. Les pointes de sagaie évoquent des chasses collectives [106], probablement lors de migrations automnales du renne, mais la plupart du temps l'acquisition s'est vraisemblablement produite sur des proies isolées. L'exploitation des ressources animales varie donc selon les régions en termes de proies et de stratégies de chasse, mais aussi dans les portions de carcasses transportées dans les sites : les segments les plus riches en viande et en graisse sont 
privilégiés, fournissant au passage des éléments propices à l'industrie osseuse en plein essor [107]. Les systèmes de mobilité semblent se rapprocher de ceux adoptés par de nombreux peuples de chasseurs-cueilleurs vivant en milieu froid, avec une relative sédentarité en abri en automne-hiver et une grande dispersion au printemps-été [105].

Autre grande civilisation du Paléolithique supérieur, le Gravettien (28-22 ka) possède des subdivisions chronoculturelles aussi complexes que son extension géographique en Europe occidentale et centrale [108]. En France, les spectres de proies étaient relativement larges et certains taxons furent privilégiés selon les régions considérées [109] : le renne prédomine le plus souvent (Périgord, Quercy), mais c'est parfois le cheval qui fut privilégié (Massif central). Cependant, une certaine variabilité des objectifs de chasse s'exprime, par exemple dans le Bassin parisien avec des chasses préférentielles aux dépend du bison (ex. site gravettien moyen des Bossats). Les profils de mortalité traduisent des stratégies diverses : le plus fréquent est de type catastrophique (équivalent à une structure de troupeau naturel), mais une sélection des adultes ou des jeunes est également documentée au Gravettien ancien et récent (sites de Solutré, Vigne-Brun, Roc de Combe, [109]). Des stratégies variées semblent avoir été élaborées pour chasser les principaux gibiers (renne, cheval, bison), permettant d'anticiper les déplacements saisonniers; en outre, le traitement boucher révèle une exploitation intensive des carcasses.

À la suite des sociétés solutréennes et badegouliennes contemporaine du Dernier Maximum Glaciaire, nous évoquerons ici le Magdalénien, dernière grande civilisation paneuropéenne. Si les premiers témoignages du Magdalénien inférieur se situent vers 17000 ans BP en Aquitaine et dans la région franco cantabrique, les derniers sites du Magdalénien supérieur-final ne vont pas au-delà de 12000 ans BP. Selon les régions, différentes stratégies d'exploitation des ressources animales sont mises en place par les groupes sociaux. Dans le Sud-Ouest de la France, la diversité des proies chassées est importante et la gamme des gibiers fluctue d'un site à l'autre [110, 111] : différents herbivores, omnivores, carnivores (par ex. lion à Garma, Espagne, [112]), mais aussi de nombreux lagopèdes et oiseaux ont été au menu ou bien ont été mis à profit à des fins techniques. Cette diversification des objectifs cynégétiques est souvent mise en relation avec des innovations techniques allant de pair avec une complexité des organisations des chasseurs-cueilleurs (sédentarité, démographie, ethnicité, etc. [113]. Le renne est néanmoins une espèce emblématique quelles que soient les régions considérées et leurs caractéristiques topographiques ou géographiques [114, 115]. Leur chasse en masse à l'automne explique souvent leur forte présence dans les spectres de chasse; cependant, les indices de saisonnalités signalent le plus souvent un abattage tout au long de l'année par les groupes magdaléniens. Dans le Bassin parisien, le cheval et le renne sont les deux proies préférentielles complémentaires [116]. Ces gibiers ont également été exploités dans différentes régions d'Allemagne et de Suisse [117, 118]. La souplesse d'une telle économie à double proie préférentielle est d'ailleurs en accord avec la recherche d'anticipation et d'efficacité, décrite pour les systèmes techniques mises en œuvre chez les Magdaléniens du Bassin parisien. Dans cette région, les chasses collectives sur ces deux proies sont communes, même si des chasses individuelles au printemps et en été aux dépend du renne sont désormais connues sur plusieurs niveaux d'occupation d'Étiolles (locus 2, Bignon-Lau, inédit). En outre, les 


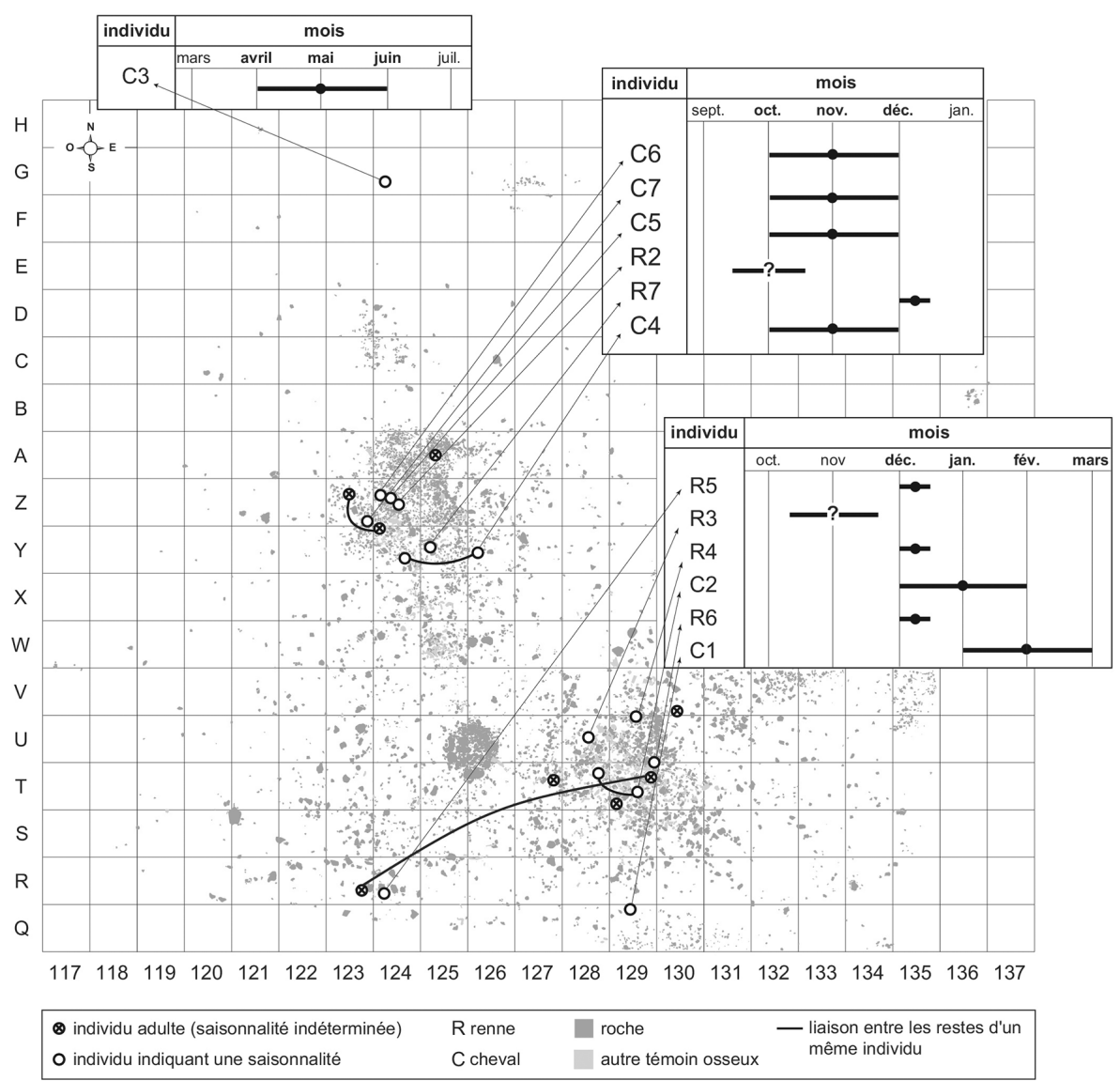

Figure 6 - Tactiques de chasse saisonnières et distribution spatiale des nappes d'ossements du niveau IV0 de Pincevent (unité T125; d'après [123]; DAO G. Debout).

sites multistratifiés de plein air comme Pincevent ou Étiolles, bénéficiant d'une conservation très favorable, permettent d'aborder les tactiques de chasse, en individualisant différents épisodes d'acquisition. Une stratégie de chasse se conçoit comme l'ensemble des dispositifs techniques (armes, installations et structuration) et les variables d'un épisode de chasse (chasse individuelle-collective, mais aussi le lieu, la saison... ). Sur le niveau IV0 de Pincevent, les rejets groupés d'ossements possédant chacun des saisons d'abattage homogènes illustrent parfaitement les objectifs cynégétiques distincts au cours du cycle annuel et leur mise en évidence par l'analyse spatiale des témoins fauniques [119] (Figure 6). Cette notion se distingue de celle de stratégie de chasse, qui renvoie à la capacité d'un groupe social à fixer et organiser des objectifs cynégétiques sur une espèce donnée au cours d'un ou plusieurs cycles annuels. Les stratégies de chasse d'un groupe humain peuvent donc se définir par les choix planifiés de l'exploitation des ressources animales (espèces, proportions relatives entre les proies, variables de classe d'âge, saisons et lieux préférentiels, etc. [116]. 


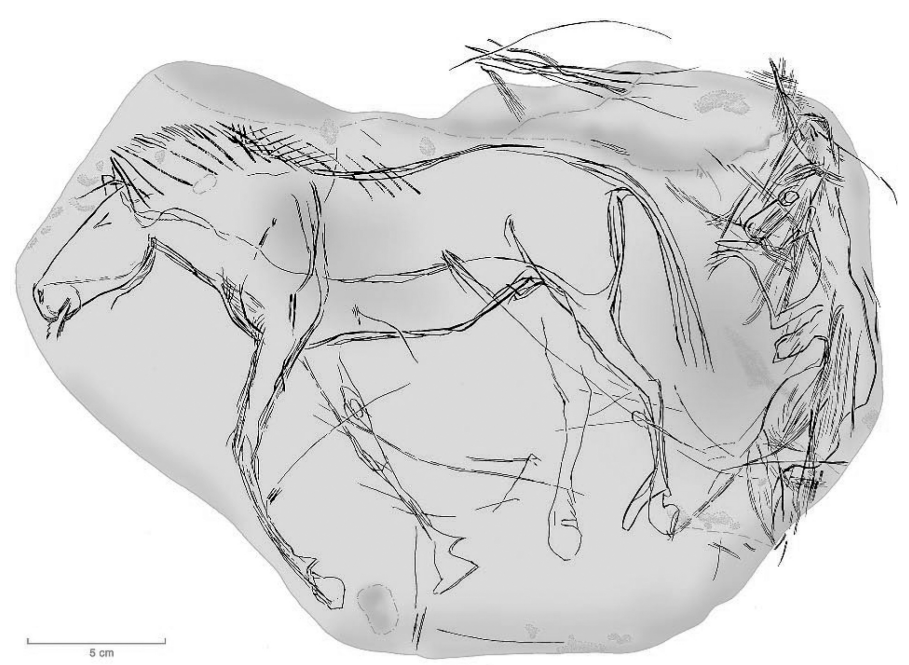

Figure 7 - Représentation du galet gravé d'Étiolles (Essonne) (d'après [124]).

En définitive, la reconnaissance de régionalismes culturels de l'Aurignacien au Magdalénien fait écho à la variabilité de l'économie des ressources animales. Celle-ci se marque dans la diversité des espèces exploitées, les stratégies de chasse, et exprime plus ou moins fidèlement une structuration variable des communautés animales au gré des paysages et des climats. Ainsi, les études morphométriques concernant le cheval [120], le renne [115] ou le mammouth [121], montrent une fragmentation régionale des populations. Mais, à travers son exploitation alimentaire, technique et les expressions esthétiques (art pariétal et mobilier; Figure 7) qui se sont développées au cours du Paléolithique supérieur, l'animal est surtout devenu un enjeu identitaire crucial à l'échelle infra-culturel pour les sociétés de chasseurs-cueilleurs.

\section{Conclusions}

Depuis près de $3 \mathrm{Ma}$, une grande diversité d'hominines s'est succédée; mais seul le genre Homo a conquis tous les continents, depuis l'Afrique, puis l'Europe, le Proche et Moyen-Orient et l'Asie, et plus récemment l'Amérique. Cette histoire traverse plusieurs périodes climatiques (phases glaciaires et interglaciaires) et des milieux très contrastés. La conquête de « nouveaux territoires » est marquée d'autant de conquêtes locales, et les dispersions humaines ont dû être très lentes et multidirectionnelles. Concomitante à l'invention de l'outil, l'apparition dans la diète de produits animaux (viande, moelle...) constitue certainement un facteur-clef dans l'émergence de nouvelles adaptations. La carnivorie, posant les questions des modes d'acquisition et de consommation, les types de gibiers, le degré de partage de nourriture, et sur les types d'actions, collectives notamment, implique le développement d'un cadre social précis et déterminé ; notion probablement centrale dans les modèles d'évolution humaine, en Afrique [125], mais aussi en Europe [126, 127]. 
Beaucoup de prospections, fouilles et études d'anciens ou de nouveaux sites archéologiques, ont été menées ces vingt dernières années et, s'il n'est pas possible de tous les citer, ces travaux ont fait avancer significativement nos connaissances sur les comportements de subsistance de sociétés humaines passées très variées. Ces nouvelles données et interprétations, ont été rendues possibles par l'apport d'outils méthodologiques multidisciplinaires, permettant de « faire parler les os » [128].

Dans ces recherches, l'apport de la taphonomie, de ses outils et problématiques, a réellement renouvelé et précisé les schémas évolutifs autant que les cadres conceptuels. Ces analyses permettent de comprendre la formation des sites, en plein air ou en grottes / abris, et de mieux interpréter les accumulations osseuses en relation avec des outillages lithiques. Il est ainsi essentiel de bien distinguer les actions relevant de l'humain de celles d'autres agents, en particulier les autres carnivores [129] ou des facteurs sédimentaires [130]. Les analyses taphonomiques ont notamment permis de préciser et même parfois de réviser de nombreuses théories, en particulier liées aux comportements de subsistance des hominines. Rappelons ici celles relatives à l'origine des ensembles osseux dans les grottes d'Afrique du Sud (remise en question de « Man the hunter »), aux mécanismes de dispersion humaine en Eurasie ( «out of Africa»), au développement des capacités d'acquisition de la viande (chasse vs charognage), dans le choix des gibiers et le traitement des carcasses (importance dans le débat entre Néandertal et Sapiens), ou encore à l'exploitation utilitaire ou symbolique des sous-produits animaux.

On assiste en effet à la complexification graduelle des comportements de subsistance des sociétés humaines au cours du temps. Si tout au long de la première période de l'évolution humaine, les proies et gibiers ont eu essentiellement un but alimentaire, l'animal est devenu peu à peu une ressource de matière première plus diversifiée, s'étendant au domaine de l'utilitaire, du rituel et du symbolique. Des stratégies d'acquisition (chasse, charognage, collecte) et des techniques de chasse (poursuite, affut, piégeage... ) variées se sont développées, en relation avec l'évolution des outillages et des armements. Les hominines ont su acquérir très tôt des produits carnés. Cette incursion ancienne dans la guilde des carnivores a sans doute été un élément moteur dans leur expansion, conduisant à des comportements socio-économiques et techniques, de plus en plus élaborés. Cette longue histoire de notre alimentation passée nous incite donc à ne pas oublier qu'encore aujourd'hui, à l'ère de la domestication et de l'élevage intensif, « nous demeurons de grands chasseurs, faits pour évoluer dans des conditions de semi-nomadisme, de faible densité démographique, dans un milieu sans inégalités socio-économiques, pour pratiquer un exercice physique intense, pour nous nourrir de gibier ou de poisson peu gras et de plantes sauvages fibreuses, sans adjonction de sel ni de toxiques divers $\gg[119: 26]$. 


\section{Remerciements}

J.-Ph. B. tient à remercier M. Dominguez-Rodrigo pour l'envoi et l'autorisation d'utiliser la Figure 2.

\section{Références}

[1] J. Ducros, A. Ducros, Le singe carnivore : la chasse chez les primates non humains. Bull. Mém. Soc. Anthrop. Paris, n.s., 4 (3-4), 243-264, 1992.

[2] B. Villmoare, W.H. Kimbel, C. Seyoum, C.J. Campisano, E.N. DiMaggio, J. Rowan, D.R. Braun, J.R. Arrowsmith, K.E. Reed, Early Homo at 2.8 Ma from Ledi-Geraru, Afar, Ethiopia. Science. 347, 1352-1355, 2016.

[3] H. Roche, R.J. Blumenschine, J.J. Shea, Origins and adaptations of early Homo : what archeology tells us. In The First Humans : Origin and Early Evolution of the Genus Homo, F.E. Grine et al. (eds.), Vertebrate Paleobiology and Paleoanthropology, C) Springer Science, 135-147; 2009.

[4] S. Harmand, J.E. Lewis, C.S. Feibel, C.J. Lepre, S. Prat, A. Lenoble, X. Boës, R.L. Quinn, M. Brenet, A. Arroyo, N. Taylor, S. Clément, G. Daver, J.P. Brugal, L. Leakey, R.A. Mortlock, J.D. Wright, D.V. Kent, H. Roche, Before the Oldowan : 3.3 Ma Stone Tools from Lomekwi 3, West Turkana, Kenya. Nature. $521: 310-315,2015$.

[5] Y. Dauphin, J.P. Brugal, Taphonomy. in Archaeology , [Eds.UNESCO-EOLSS Joint Commitee] ,in Encyclopedia of Life Support Systems(EOLSS), Developed under the auspices of the UNESCO, Eolss Publishers, Oxford ,UK, [http ://www.eolss.net], 2013.

[6] C.K. Brain, 1981. The hunters or the hunted? An introduction to African cave taphonomy. Ed. Univ. Chicago Press, 365 pp., 1981.

[7] A.K. Behrensmeyer, A. Hill, Fossils in the Making. Ed. Univ. Chicago Press, 345 p., 1981

[8] G.L. Isaac, The food-sharing behavior of protohuman hominids. Scientific American. 238, 90-108, 1978.

[9] G.L. Isaac, Bones in contention : competing explanations for the juxtaposition of Early Pleistocene artifacts and faunal remains. In Animals and Archaeology 1. Hunters and Their Prey, eds. J. CluttonBrock, C. Grigson, BAR International Series 163, Oxford, pp. 3-19, 1983.

[10] G.L. Isaac, The archaeology of human origins : studies of Lower Pleistocene in East Africa, $1971-1981$. Advances in World Archaeology. 3, 1-87, 1984.

[11] L.R. Binford, Bones : Ancient Men. Modern Myths. Academic Press, New York. 1981.

[12] L.R. Binford, Human ancestors : changing views of their behavior. Journal of Anthropological Archaeology. 4, 292-327, 1985.

[13] J.-P. Brugal, Eco-éthologie des premiers hominidés en Afrique : les activités de subsistance. Bull. Mém. Soc. Anthrop. Paris , n.s., 4 (3-4), 143-166, 1992.

[14] S.P. McPherron, Z. Alemseged, C.W. Marean, J.G. Wynn, D. Reed, D. Geraads, R. Bobe, A.H. Be'arat. Evidence for stone-tool-assisted consumption of animal tissues before 3.39 million years ago at Dikika, Ethiopia. Nature. 466, 857-860, 2010.

[15] M. Domínguez-Rodrigo, T.R. Pickering, S. Sileshi Semaw, M.J. Rogers, Cutmarked bones from Pliocene archaeological sites at Gona, Afar, Ethiopia : implications for the function of the world's oldest stone tools. Journal of Human Evolution. 48, 109-121, 2005.

[16] J.J. Tiercelin, M. Schuster, H. Roche, J.P. Brugal, P. Thuo, S. Prat, S. Harmand, G. Davtian, J.A. Barrat, M. Bohn, New considerations on the stratigraphy and environmental context of the oldest (2.34 Ma) Lokalalei archaeological site complex of the Nachukui Formation, West Turkana, northern Kenya Rift, Journal of African Earth Sciences. 58(2), 157-184, 2010.

[17] J.M. Harris, F.H. Brown, M.G. Leakey, Stratigraphy and paleontology of Pliocene and Pleistocene localities west of Lake Turkana, Kenya. Ed.Natural History Museum of Los Angeles County, 1988.

[18] J.P. Brugal, H. Roche, M. Kibunjia M. 2003. Faunes et paléoenvironnements des principaux sites archéologiques plio-pleistocènes de la formation de Nachukui (Ouest Turkana, Kenya). C. R. Palevol. 2(8), 675-684, 2003.

[19] J.V. Ferraro, T.W. Plummer, B.L. Pobiner, J.S. Oliver, L.C. Bishop, D.R. Braun, P.W. Ditchfield, J.W. III Seaman, K.M. Binetti, J.W. Jr. Seaman, F. Hertel, R. Potts, Earliest archaeological evidence of persistent Hominin carnivory. PlosOne. 8(4), e62174, 2013. 
[20] D.R Braun., J.W.K. Harris, N.E Levin., J.T. McCoy, A.I.R. Herries, M.K. Bamford, L.C. Bishop, B.G.Richmond, M.Kibunjia, Early hominin diet included diverse terrestrial and aquatic animals $1.95 \mathrm{Ma}$ in East Turkana, Kenya. PNAS. 107(22), 10002-10007, 2010.

[21] R.J. Blumenshine, Carcass consumption sequence and the archaeological distinction of scavenging and hunting. Journal of Human Evolution. 15, 639-659, 1986.

[22] R.J. Blumenschine, C.R. Peters, F.T. Masao, R.J. Clarke, A.L. Deino, R.L. Hay, C.C. III Swisher, I.G. Stanistreet, G.M. Ashely, L. McHenry, N.E. Sikes, N.J. Van der Merwe, J.C. Tactikos, A.E. Cushing, D.M. Deocampo, J.K. Njau, J.J. Ebert, Late Pliocene Homo and hominid land use from Western Olduvai Gorge, Tanzania. Science. 299, 1217-1221, 2003.

[23] M. Domínguez-Rodrigo, Are all Oldowan Sites Palimpsests? If so, what can they tell us about Hominid Carnivory? In Interdisciplinary Approaches to the Oldowan, eds. E. Hovers, D.R. Braun, (C) Springer Science, 129-147, 2009.

[24] M. Domínguez-Rodrigo, R. Barba Egido, C.P. Egeland, Deconstructing Olduvai : a taphonomic study of the Bed I sites. Vertebrate Paleobiology and Paleoanthropology series, Springer Verlag, 337 p., 2007.

[25] E. Boëda, Y.M. Hou, W.-B. Huang, Introduction à l'étude du site de Longgupo. L'Anthropologie. $115(1), 8-22,2011$.

[26] M. Tappen, D. Lordkipanidze, M. Bukshianidze, R. Ferring, A. Vekua, Are you in or out (of Africa)? site formation at Dmanisi and actualistic studies in Africa. In Breatthing Life into Fossils : Taphonomic Studies in Honor of C.K. (Bob) Brain. Eds. T.R. Pickering, K. Schick, N. Toth, Stone Age Institute Publication Series, Gosport (Indiana), 2 : 119-135, 2007.

[27] G. Landeck, J. Garcia Garriga, The oldest hominin butchery in European mid-latitudes at the Jaramillo site of Untermassfeld (Thuringia, Germany). Journal of Human Evolution. 94, 53-71, 2016.

[28] L. Bourguignon, J.Y. Crochet, R. Capdevila, J. Ivorra, P.-O. Antoine, J. Agustí, D. Barsky, H.-A. Blain, N. Boulbes, L. Bruxelles, J. Claude, D. Cochard, A. Filoux, C. Firmat, I. Lozano-Fernández, P. Magniez, M. Pelletier, J. Rios-Garaizar, A. Testu, P. Valensi, L. De Weyer, Bois-de-Riquet (Lézignan-la-Cèbe, Hérault) : A late Early Pleistocene archeological occurrence in southern France. Quaternary International. 393, 2440, 2016.

[29] M.P. Espigares, B. Martínez-Navarro, P. Palmqvist, S. Ros-Montoya, I. Toro, J. Agustí, R. Sala, Homo vs. Pachycrocuta : Earliest evidence of competition for an elephant carcass between scavengers at Fuente Nueva-3 (Orce, Spain). Quaternary International. 295, 113-125, 2013.

[30] R. Huguet, J. Vallverdú, X.P. Rodríguez-Alvarez, M. Terradillos-Bernal, A. Bargalló, A. LomberaHermida, L. Menéndez, M. Modesto-Mata, J. Van der Made, M. Soto, H.-A. Blain, N. García, G. CuencaBescó, G. Gómez-Merino, R. Pérez-Martínez, I. Expósito, E. Allué, J. Rofes, F. Burjachs, A. Canals, M. Bennàsar, C. Nunez-Lahuerta, J.M. Bermúdez de Castro, E. Carbonell, Level TE9c of Sima del Elefante (Sierra de Atapuerca, Spain) : A comprehensive approach. Quaternary International. http ://dx.doi.org/10. 1016/j.quaint.2015.11.030

[31] J.-P. Brugal, J. Jaubert, Les gisements paléontologiques pléistocènes à indices de fréquentation humaine : un nouveau type de comportement prédateur? Paleo. 3, 1-5, 1991.

[32] J.C. Díez, Y. Fernández-Jalvo, I. Cáceres, J. Rosell, Zooarchaeology and taphonomy of Aurora stratum (Gran Dolina, Sierra de Atapuerca, Spain). Journal of Human Evolution. 37, 623-657, 1999.

[33] A. Bouteaux, A.M. Moigne, New taphonomical approaches : The Javanese Pleistocene open-air sites (Sangiran, central Java). Quaternary International. 223-224, 220-225, 2010.

[34] N. Goren-Inbar, A. Lister, E. Werker, M. Chech, A butchered elephant skull and associated artifacts from the Acheulian site of Gesher Benot Ya'aqov, Israel. Paléorient. 20, 99-112, 1994.

[35] A.M. Moigne, L'étude des vestiges osseux du gisement acheuléen de Cagny l'Epinette (Somme). Revue archéologique de Picardie. 1-2, 69-71, 1988.

[36] U. Thun Hohenstein, A. Di Nucci, A.M. Moigne, Mode de vie à Isernia La Pineta (Molise, Italie). Stratégie d'exploitation du Bison schoetensacki par les groupes humains au Paléolithique inférieur. $L$ 'Anthropologie. 113, 96-110, 2009.

[37] F. Rivals, A. Testu, A.M. Moigne, H. de Lumley, The Middle Pleistocene argali (Ovis ammon antiqua) assemblages at the Caune de l'Arago (Tautavel, Pyrénées-Orientales, France) : were prehistoric hunters or carnivores responsible for their accumulation? International Journal of Osteoarchaeology. 16, 249-268, 2006.

[38] J.J. Hublin, The origin of Neandertals. Proceedings of the National Academy of Sciences. 106 (38), 16022-16027, 2009. 
[39] P. Endicott, S.Y.W. Ho, C. Stringer, Using genetic evidence to evaluate four palaeoanthropological hypotheses for the timing of Neanderthal and modern hominin origins. Journal of Human Evolution. 59, 87-95, 2010.

[40] R-D. Kahlke, N. Garcia, D.S. Kostopoulos, F. Lacombat, A.M. Lister, P. Mazza, N. Spassov, V.V. Titov, Western Palaearctic palaeoenvironmental conditions during the Early and early Middle Pleistocene inferred from large mammal communities, and implications for hominin dispersal in Europe. Quaternary Science Reviews. 30, 1368-1395, 2011.

[41] J.P. Brugal, P. Fosse, Carnivores et Hommes au Quaternaire en Europe de l'Ouest. Revue de Paléobiologie. Genève, 23 (2), 575-595, 2004.

[42] H. Thieme, Lower Palaeolithic hunting spears from Germany. Nature. 385, 805-810, 1997.

[43] K.P. Oakley, P. Andrews, L.H. Keeley, J.D. Clark, A reappraisal of the Clacton spearpoint. Proceedings of Prehistoric Society. 43, 13-30, 1977.

[44] S. Gaudzinski, Subsistence patterns of Early Pleistocene hominids in the Levant- taphonomic evidence from the 'Ubeidiya Formation (Israel). Journal of Archaeological Science. 31, 65-75, 2004.

[45] M.H. Moncel, A.M. Moigne, J. Combier, Towards the Middle Palaeolithic in Western Europe : The case of Orgnac 3 (southeastern France). Journal of Human Evolution. 6, 653-666, 2012.

[46] A. Turq, M. Brenet, D. Colonge, M. Jarry, L.A. Lelouvier, M. O'Farrell, J. Jaubert, The first human occupations in Southwestern France : a revised summary twenty years after the Abbeville/Saint Riquier Colloquium. Special issue, human expansions in Eurasia. Quaternary International. 223 224, 383-398, 2010 .

[47] W. Roebroeks, P. Villa, On the earliest evidence for habitual use of fire in Europe. Proceedings of the National Academy of Sciences. 108 (13), 5209-5214, 2011.

[48] P. Auguste, Chasse et charognage au Paléolithique moyen : l'apport du gisement de Biache-Saint-Vaast (Pas-de-Calais). Bulletin de la Société Préhistorique Française. 92 (2), 155-167, 1995.

[49] P. Valensi, « Les grands mammifères de la grotte du Lazaret, Nice - Etude paléontologique et biostratigraphique des carnivores, archéozoologie des grandes faunes ». Thèse de Doctorat, MNHN, Paris, 1994.

[50] J. Jaubert, J.P. Brugal J.-P., Contribution à l'étude du mode de vie au Paléolithique moyen : les chasseurs d'Aurochs de La Borde. in Les chasseurs d'Aurochs de La Borde. Un site du Paléolithique moyen (Livernon, Lot), J.Jaubert et al., Paris : Maison des Sciences de l'Homme, Documents d'Archéologie française, 27, 128-145, 1990.

[51] J. Jaubert, B. Kervazo, J.-P. Brugal, C. Falgueres, M. Jeannet, A. Louchard, H. Martin, F. Maksud, V. Mourre, Y. Quinif, La séquence Pléistocène moyen de Coudoulous I (Lot). Bilan pluridisciplinaire. In Les premiers peuplements en Europe: Données récentes sur les modalités de peuplement et sur le cadre chronostratigraphique, géologique et paléogéographique des industries du Paléolithique ancien et moyen en Europe, eds. N. Molines, M.H. Moncel, J.L. Monnier, Oxford, British Archaeological Reports International Series $1364: 237-251,2005$.

[52] R. Blasco, J. Rosell, Who was the first? An experimental application of carnivore and hominid overlapping marks at the Pleistocene archaeological sites. C. R. Palevol. 8, 579-592, 2009.

[53] H.R. Pamies, C. Diez Fernandez-Lomana, J. Rosell Ardevol, I. Caceres Cuello De Oro I., V. Moreno Lara, N. Ibanez Lopez, P. Saladie Balleste P., Le gisement de Galeria (Sierra de Atapuerca, Burgos, Espagne) : un modèle archéozoologique de gestion du territoire au Pléistocène. L'Anthropologie. 105, 237-257, 2001.

[54] M.H. Moncel (dir.), Le site de Payre. Occupations humaines dans la moyenne vallée du Rhône à la fin du Pléistocène moyen et au début du Pléistocène supérieur. Société Préhistorique Française. Mémoire 46, 336 p., 2008.

[55] C. Daujeard, M.H. Moncel, P. Auguste, D. Aureli, H. Bocherens, E. Cregut-bonnoure, E. Debard, M. Liouville, P. Fernandez, F. Rivals, Quel type d'occupation dans l'ensemble F de Payre (Ardèche, France)? Halte de chasse spécialisée ou campement de courte durée? Un exemple d'approche multi disciplinaire. P@lethnologie. 2011.

[56] J.P. Brugal, C. Diez-Lomana, R. Huguet Pamies, P. Michel, J. Rosell Ardevol, Karstic cavities, natural bone accumulations and discrete human activities in the European Palaeolithic : some case studies. In Paleolithic zooarchaeology in practice, eds. J. Haws, J.P. Brugal, B. S. Hockett, Oxford, British Archaeological Reports International Series 1564, 1-12, 2006. 
[57] R. Blasco, J. Fernández Peris., J. Rosell, Several different strategies for obtaining animal resources in the late Middle Pleistocene: The case of level XII at Bolomor Cave (Valencia, Spain). C. R. Palevol. 9, 171-184, 2010.

[58] P. Valensi, E. Psathi, Faunal exploitation during the Middle Palaeolithic in south-eastern France and north-western Italy. International Journal of Osteoarchaeology. 14, 256-272, 2004.

[59] J. Serangeli, T. van Kolfschoten, B.M. Starkovich, I. Verheijen, The European saber-toothed cat (Homotherium latidens) found in the "Spear Horizon" at Schöningen (Germany). Journal of Human Evolution. 89, 172-180, 2015.

[60] P. Villa, E. Soto, M. Santonja, A. Perez-Gonzalez, R. Mora, J. Parcerisas, C. Sese, New data from Ambrona : closing the hunting versus scavenging debate. Quaternary International. 126-128, 223-250, 2005.

[61] J. Yravedra, M. Domínguez-Rodrigo, M. Santonja M., A. Pérez-González, J. Panera, S. Rubio-Jara, E. Baquedano, Cut marks on the Middle Pleistocene elephant carcass of Áridos 2 (Madrid, Spain). Journal of Archaeological Science. 37, 2469-2476, 2010.

[62] E. Santucci, F. Marano, E. Cerilli, I. Fiore, C. Lemorini, M.R. Palombo, A.P. Anzidei, G.M. Bulgarelli, Palaeoloxodon exploitation at the Middle Pleistocene site of La Polledrara di Cecanibbio (Rome, Italy). Quaternary International. 406, 169-182, 2016.

[63] G. Smith, Neanderthal megafaunal exploitation in Western Europe and its dietary implications : A contextual reassessment of La Cotte de St Brelade (Jersey). Journal of Human Evolution. 78, 181-201, 2015.

[64] M.C. Stiner, A. Gopher, R. Barkai, Cooperative hunting and meat sharing 400-200 kya at Qesem Cave, Israel. PNAS. 106 (32), 13207-13212, 2009.

[65] C. Gamble, W. Roebroeks, The Middle Palaeolithic : a point of inflexion. in The Middle Palaeolithic Occupation of Europe, eds. W. Roebroeks, C. Gamble, University of Leiden, p. 3-21, 1999.

[66] L.R. Binford, Isolating the transition to cultural adaptations : an organizational approach. In The emergence of modern humans. Biocultural adaptations in the Late Pleistocene. Ed. E. Trinkaus, Cambridge University Press, 18-41, 1989.

[67] P.G. Chase, How different was Middle Palaeolithic subsistence? A zoological perspective on the Middle to Upper Palaeolithic transition. In The Human Revolution : Behavioural and biological perspectives in the Origins of Modern Humans. Eds. P. Mellars, C. Stringer C., Edinburgh University Press, Edinburgh, 321-337, 1989.

[68] C. Daujeard, M.-H. Moncel, On Neanderthal subsistence strategies and land use : A regional focus on the Rhone Valley area in southeastern France. Journal of Anthropological Archaeology. 29, 368-391, 2010.

[69] J. Marín, P. Saladié, A. Rodríguez-Hidalgo, E. Carbonell, Ungulate carcass transport strategies at the Middle Palaeolithic site of Abric Romani (Capellades, Spain). C.R. Palevol. http ://dx.doi.org/10.1016/j.cr pv.2015.11.006, ss-pr.

[70] E. Morin, Reassessing Paleolithic Subsistence. The Neandertal and Modern Human foragers of SaintCésaire. Cambridge University Press. 358 p., 2012.

[71] J. Rossel, I. Cáceres, R. Blasco, M. Bennàsar, P. Bravo, G. Campeny, M. Esteban-Nadal, M. C. Fernández-Laso, M. J. Gabucio, R. Huguet, N. Ibáñez, P. Martín, F. Rivals, A. Rodríguez-Hidalgo, P. Saladié, A zooarchaeological contribution to establish occupational patterns at Level J of Abric Romani (Barcelona, Spain). Quaternary International. 247, 69-84, 2012.

[72] W. Rendu, Hunting behavior and Neanderthal adaptability in the Late Pleistocene site of Pech-de-l'Azé I. Journal of Archaeological Science. 37, 1798-1810, 2010.

[73] I. Fiore, M. Gala, A. Tagliacozzo, Ecology and subsistence strategies in the eastern Italian Alps during the Middle Palaeolithic. International Journal of Osteoarchaeology. 14, 273-286, 2004.

[74] A. Gardeisen, Middle palaeolithic subsistence in the West Cave of "Le Portel" (Pyrénées, France). Journal of Archaeological Science. 26, 1145-1158, 1999.

[75] M. Patou-Mathis, Etude taphonomique et palethnographique de la faune de l'abri des Canalettes. In L'abri des Canalettes. Un habitat moustérien sur les Grands Causses (Nant, Aveyron). Ed. L. Meignen, Paris, CNRS Editions. 199-237, 1993.

[76] M. Patou-Mathis, Neanderthal, une autre humanité. Perrin, Tempus, 376 p., 2006.

[77] J. Yravedra, L. Cobo-Sánchez, Neanderthal exploitation of ibex and chamois in southwestern Europe. Journal of Human Evolution. 78, 12-32, 2015. 
[78] S. Costamagno, L. Meignen, C. Beauval, B. vandermeersch, B., Maureille, Les Pradelles (Marillacle-Franc, France) : A Mousterian reindeer hunting camp? Journal of Anthropological Archaeology. 25, 466-484, 2006.

[79] W. Rendu, S. Costamagno, L. Meignen, M.-C. Soulier, Monospecific faunal spectra in Mousterian contexts : Implications for social behavior. Quaternary International. 247, 50-58, 2012.

[80] S. Gaudzinski, W. Roebroeks, Adults only. Reindeer hunting at the Middle Palaeolithic site Salzgitter Lebenstedt, Northern Germany. Journal of Human Evolution. 38, 497-521, 2000.

[81] L. Niven, T.E. Steele, W. Rendu, J.B. Mallye, S.P. McPherron, M. Soressi, J. Jaubert, J.-J. Hublin, Neandertal mobility and large-game hunting : the exploitation of reindeer during the Quina Mousterian at Chez-Pinaud Jonzac (Charente-Maritime, France). Journal of Human Evolution. 63, 624-635, 2012.

[82] C. Farizy, F. David, J. Jaubert, Hommes et bisons du Paléolithique moyen à Mauran (Haute-Garonne). CNRS, XXX ${ }^{\circ}$ supplément à Gallia Préhistoire, Paris, 1994.

[83] F. David, P. Fosse, Le bison comme moyen de subsistance au Paléolithique : gisements de plein air et sites en grotte. In Le Bison : gibier et moyen de subsistance des hommes du Paléolithique aux paléoindiens des grandes plaines. Eds. J.P. Brugal, F. David, J.G. Enloe, J. Jaubert J., Actes du colloque international, Toulouse 1995. Editions APDCA, Antibes. 121-141, 1999.

[84] R. Blasco, J. Fernández Peris, A uniquely broad spectrum diet during the Middle Pleistocene at Bolomor Cave (Valencia, Spain). Quaternary International. 252, 16-31, 2012.

[85] B.L. Hardy, M.-H. Moncel, Neanderthal use of fish, mammals, birds, starchy plants and wood 125250,000 years ago. PLoS One. 6, e23768, 2011.

[86] M. Peresani, I. Fiore, M. Gala, M., Romandini, A. Tagliacozzo, Late Neandertals and the intentional removal of feathers as evidenced from bird bone taphonomy et Fumane Cave 44 ky B.P., Italy. PNAS. 108, 3888-3893, 2011.

[87] D. Cochard, J.-P., Brugal, E. Morin, L. Meignen, Evidence of small fast game exploitation in the Middle Paleolithic of Les Canalettes Aveyron, France. Quaternary International. 264, 32-51, 2012.

[88] S. Costamagno, C. Beauval, B. Lange-Badré, B. Vandermeersch, A. Mann, B. Maureille, Homme ou carnivores? Protocole d'étude d'ensembles osseux mixtes : l'exemple du gisement moustérien des Pradelles (Marillac-le-Franc, Charente). Archaeofauna. 14, 43-68, 2005.

[89] J.G. Enloe, Middle Palaeolithic Cave taphonomy : Discerning humans from hyenas at Arcy-sur-Cure, France. International Journal of Osteoarchaeology. 22, 591-602, 2011.

[90] J.-P. Brugal, P. Fosse, J.-L. Guadelli, Comparative study of bone assemblages made by recent and Pleistocene hyenids. In Proceedings of the 1993 Bone modification conference, eds. L.A. Hannus, L. Rossum, R.P. Winham, Hot Springs, South Dakota. Occasional publication $\mathrm{N}^{\circ} 1$, Archaeology Laboratory, Augusta College, Sioux Falls, 158-187, 1997.

[91] T.R. Pickering, Reconsideration of criteria for differentiating faunal assemblages accumulated by hyenas and hominids. International of Osteoarchaeology. 12, 127-141, 2002.

[92] P. Magniez, Taphonomic study of the middle and upper palaeolithic large mammal assemblage from Tournal Cave (Bize-Minervois, France). Journal of Taphonomy. 7, 203-233, 2009.

[93] G. Abrams, S.M. Bello, K. Di Modica, S. Pirson, D. Bonjean, When Neanderthals used cave bear (Ursus spelaeus) remains : Bone retouchers from unit 5 of Scladina Cave (Belgium). Quaternary International. 326/327, 274-287, 2014.

[94] C. Daujeard, M.-H. Moncel, I. Fiore, A. Tagliacozzo, P. Bindon, J.-P. Raynal, Middle Paleolithic bone retouchers in Southeastern France : Variability and functionality. Quaternary International. 326/327, 492$518,2014$.

[95] A. Svensson, K. K. Andersena, M. Biglera, H. B. Clausena, D. Dahl-Jensena, S. M. Daviesb, S. J. Johnsena, R. Muschelerc, S. O. Rasmussena, R. Röthlisbergerd, J. P. Steffensena, B. M. Vinther, The Greenland Ice Core Chronology 2005, 15-42 ka. Part 2 : comparison to other records. Quaternary Science Reviews. 25, 3258-3267, 2006.

[96] M.F. Sanchez-Goñi, S. P. Harrison, Millennial-scale climate variability and vegetation changes during the Last Glacial : Concepts and terminology. Quaternary Science Reviews. 29, 2823-2827, 2010.

[97] F. d'Errico, J. Zilhao, M. Julien, D. Baffier, J. Pelegrin, Neanderthal acculturation in Western Europe? Acritical review of the evidence and its interpretation. Current Anthropology, 39, 1-44, 1998.

[98] F. Bon, L'Aurignacien entre Mer et Océan. Réflexion sur l'unité des phases anciennes de l'Aurignacien dans le Sud de la France, Société préhistorique française, Mémoire 24, Paris, 253 p., 2002. 
[99] J. Zilhão, F. d'Errico (eds), The chronology of the Aurignacian and of the transitional technocomplexes. Where do we stand?. Instituto Portuguese de Arqueologia, Lisbonne, 313- 349, 2003.

[100] N. Teyssandier, F. Bon, J.-G. Bordes, Within Projectile Range. Some Thoughts on the Appearance of the Aurignacian in Europe. Journal of Anthropological Research. 66, 209-229, 2010.

[101] V. Guillomet-Malmassari, D'une révolution à l'autre. Pour une épistémologie de la problématique de transition en Préhistoire. Société préhistorique française. Mémoire 52, 132 p., 2012.

[102] R.D. Guthrie, Frozen Fauna of the Mammoth Steppe : The Story of the Blue Babe, University of Chicago Press, Chicago, 1990.

[103] M.-P. Coumont, Les avens-pièges. Taphonomie et paléoécologie d'accumulations naturelles fossiles de faune. Apports pour la compréhension des archéofaunes. Bulletin de la Société préhistorique française. 105(4), 691-707, 2008.

[104] A.D. Barnosky, P.L. Koch, R.S. Feranec, S.L. Wing, A.B. Shabel, Assessing the causes of Late Pleistocene extinctions on the continents. Science. 306, 70-75, 2004.

[105] M.-C. Soulier, « Entre alimentaire et technique : l'exploitation animale aux débuts du Paléolithique supérieur. Stratégies de subsistance et chaînes opératoires de traitement du gibier à Isturitz, La Quina aval, Roc-de-Combe et Les Abeilles », thèse de doctorat de l'université de Toulouse II-Le Mirail, 756 p., 2013.

[106] J. Pelegrin, Les techniques de débitage laminaire au Tardiglaciaire : critères de diagnose et quelques réflexions. (eds) B. Valentin, P. Bodu, M. Christensen, Actes de la Table ronde internationale de Nemours (14-15-16 mai 1997), Éditions APRAIF, Mémoire du musée de Préhistoire d'Île-de-France $\mathrm{n}^{\circ} 7$, Nemours, 73-86, 2000.

[107] E. Tartar, « De l'Os à l'Outil : Caractérisation technique, économique et sociale de l'utilisation de l'os à l'Aurignacien ancien. Étude de trois sites : l'Abri Castanet (secteurs nord et sud), Brassempouy (Grotte des Hyènes et Abri Dubalen) et Gatzarria », thèse de doctorat de l'université de Paris 1- Panthéon-Sorbonne, 2009 .

[108] L. Klaric, «Peut-on dresser un cadre chrono-culturel fiable pour le Gravettien du Bassin parisien?» (eds.) P. Bodu, L. Chehmana, L. Klaric, L. Mevel, S. Soriano, N. Teyssandier, Société préhistorique française. Mémoire 54, Paris, 61-88, 2013.

[109] J. Lacarrière, « Les ressources cynégétiques au Gravettien en France. Acquisition et modalités d'exploitation des animaux durant la phase d'instabilité climatique précédant le dernier maximum glaciaire », thèse de doctorat de l'université de Toulouse II-Le Mirail, 468 p., 2015.

[110] F. Delpech, Les faunes du Paléolithique supérieur dans le Sud-Ouest de la France. CNRS Éditions Cahier du Quaternaire n 6, Paris, 453 p., 1983.

[111] S. Costamagno, «Stratégies de chasse et fonction des sites au Magdalénien dans le Sud de la France », thèse de doctorat de l'université de Bordeaux I, 2 volumes, 479 p., 1999.

[112] M. Cueto, E. Camarós, P. Castaños, R. Ontañón, P. Arias, Under the Skin of a Lion : Unique Evidence of Upper Paleolithic Exploitation and Use of Cave Lion (Panthera spelaea) from the Lower Gallery of La Garma (Spain). PloSone, http ://dx.doi.org/10.1371/journal.pone.0163591, 2016.

[113] D. Cochard, J.-P. Brugal, Importance des fonctions de sites dans les accumulations paléolithiques de léporidés, Actes des XXIVe rencontres internationales d'archéologie et d'histoire d'Antibes, eds. J.P. Brugal, J. Desse, Éditions APDCA, Antibes, 283-296, 2004.

[114] L. Fontana, « Mobilité et subsistance au Magdalénien dans le Languedoc occidental et le Roussillon », thèse de Préhistoire-Ethnologie-Anthropologie de l'université de Paris 1 - Panthéon-Sorbonne, 576 p., 1998.

[115] D. Kuntz, « Ostéométrie et migration(s) du Renne (Rangifer Tarandus) dans le sud-ouest de la France au cours du dernier Pléniglaciaire et du Tardiglaciaire (21 $500-13000$ cal. BP) », thèse de doctorat de l'université de Toulouse II-Le Mirail, 496 p., 2011.

[116] O. Bignon, Chasser les chevaux à la fin du Paléolithique dans le Bassin parisien. Stratégie cynégétique et mode de vie au Magdalénien et à l'Azilien ancien. Oxford, BAR International Series 1747, 170 p., 2008.

[117] G.C. Weniger, The Magdalenian in Western Central Europe : Settlement pattern and regionality. Journal of World Prehistory. 3 : 323-372, 1989.

[118] P. Morel, W. Müller, Hauterive-Champréveyres, 11. Un campement magdalénien au bord du lac de Neuchâtel : étude archéozoologique (secteur 1). Musée cantonal d'archéologie, Archéologie neuchâteloise, 23, Neuchâtel, 149 p., 1997. 
[119] O. Bignon-Lau, Hunting practices targeting Large mammals communities of the Paris basin during the Upper Paleolithic. Quaternary International. 337, 114-128, 2014.

[120] O. Bignon, M. Baylac, J.-D. Vigne, V. Eisenmann, Geometric morphometrics and the population diversity of Late Glacial horses in Western Europe (Equus caballus arcelini) : phylogeographic and anthropological implications. Journal of Archaeological Science. 32, 375-391, 2005.

[121] A.M. Lister, A.V. Sher, The Origin and Evolution of the Woolly Mammoth. Science. 294, 1094-1097, 2001.

[123] G. Debout, O. Bignon, J.G. Enloe, Répartition des témoins animaux : une gestion de l'espace rythmée par les saisons?, (eds) P. Bodu, M. Julien, B. Valentin, G. Debout, Gallia Préhistoire. 48, 134-135, 2006.

[124] C. Fritz, G. Tosello, Exceptional evidence for Palaeolithic art in the Paris Basin : the engraved pebble from Étiolles (Essonne). Bulletin de la Société préhistorique française. 108(1), 27-46, 2011.

[125] R. Potts, Early hominid activities at Olduvai. Aldine de Gruyter, New-York, 1988.

[126] M.C. Stiner, Honor among thieves : A zooarchaeological perspective on Neandertal ecology. Princeton University Press, 1994.

[127] J.P. Brugal, L. Meignen, M. Patou-Mathis (éds.), Economie Préhistorique : les comportements de subsistance au Paléolithique. XVIIIe Rencontres Internationales d'Archéologie et d'Histoire d'Antibes, Sophia Antipolis : éd. APDCA, 467 p., 1998.

[128] M. Balasse, J.P. Brugal, Y. Dauphin, E.M. Geigl, C. Oberlin, I. Reiche I. (coord), Messages d'Os. Archéométrie du squelette animal et humain. Ed. Archives Contemporaines, coll. Sciences Archéologiques, 530 p., 2015.

[129] J.B. Fourvel, Accumulations par les prédateurs non-humains. ce vol., 2017.

[130] P. Bertran, D. Todisco, J.G. Bordes, L. Vallin, Géoarchéologie et taphonomie des vestiges archéologiques : impacts des processus naturels sur les assemblages et méthodes d'analyse. ce vol., 2017.

[131] G. Delluc avec B. Delluc, M. Roques, La nutrition préhistorique. Périgueux, Pilote 24, 223 p., 1995. 